\title{
Hypoxic pulmonary vasoconstriction disappears in a rabbit model of cavopulmonary shunt
}

\author{
Akio Ikai, MDa \\ Mikiyasu Shirai, MD, $\mathrm{PhD}^{\mathrm{b}}$ \\ Kazunobu Nishimura, MD, $\mathrm{PhD}^{\mathrm{a}}$ \\ Tadashi Ikeda, MD, PhD ${ }^{a}$ \\ Takayuki Kameyama, MD \\ Koji Ueyama, MD \\ Masashi Komeda, MD, PhD
}

From the Department of Cardiovascular Surgery, Graduate School of Medicine, Kyoto University, Kyoto, Japan, and the Department of Cardiac Physiology, National Cardiovascular Center Research Institute, ${ }^{\text {b }}$ Osaka, Japan

Received for publication March 2, 2003; revisions requested April 11, 2003; revisions received June 13, 2003; accepted for publication July 7, 2003.

Address for reprints: Masashi Komeda, $\mathrm{MD}, \mathrm{PhD}$, Kyoto University, Graduate School of Medicine, Department of Cardiovascular Surgery, 54 Shogoinkawahara-cho Sakyo-ku, Kyoto, Japan, 606-8507 (E-mail: masakom@kuhp.kyoto-u.ac.jp).

J Thorac Cardiovasc Surg 2004;127:1450-7

$0022-5223 / \$ 30.00$

Copyright (C) 2004 by The American Association for Thoracic Surgery

doi:10.1016/S0022-5223(03)01191-7
Background: Cavopulmonary shunt is widely known as an interim staging procedure in patients with single-ventricle physiology. However, the physiologic characteristics of the pulmonary arterial system after cavopulmonary shunt are not clearly understood. In this article, we developed a rabbit cavopulmonary shunt model and studied the morphologic changes and physiologic characteristics (namely, hypoxic pulmonary vasoconstriction) of pulmonary arteries after cavopulmonary shunt.

Methods: Male Japanese white rabbits aged 12 to 16 weeks were used for the study. In 5 rabbits, the superior vena cava was anastomosed to the right pulmonary artery in an end-to-side fashion, followed by a proximal side ligation of the right pulmonary artery (cavopulmonary shunt group). In 4 rabbits, the superior vena cava and the right pulmonary artery were dissected and clamped for 10 minutes without making a cavopulmonary shunt (sham group). Two weeks after the operation, we then measured the internal diameter of the acinar (internal diameter, $164 \pm 7 \mu \mathrm{m}$ ), the lobular $(305 \pm 13 \mu \mathrm{m})$, and the segmental $(669 \pm 16 \mu \mathrm{m})$ pulmonary arteries in both controlled and hypoxic conditions by using a specially designed $\mathrm{x}$-ray television system. Also, morphometric measurements were made in the pulmonary arteries around the terminal bronchioles.

Results: Two weeks after the operation, the arterial oxygen tension under room air conditions was significantly lower in the cavopulmonary shunt group than in the sham group $(68.2 \pm 2.2 \mathrm{~mm} \mathrm{Hg}$ vs $91.1 \pm 1.9 \mathrm{~mm} \mathrm{Hg} ; P=.01)$. The baseline internal diameters in the acinar and the lobular (resistance), but not the segmental (conduit), pulmonary arteries on the anastomosed side of the cavopulmonary shunt group were significantly larger than those of pulmonary arteries on the nonanastomosed side of the cavopulmonary shunt group and the sham group. Moreover, the pulmonary arteries on the anastomosed side of the cavopulmonary shunt group did not respond to hypoxia, whereas those on the nonanastomosed side of the cavopulmonary shunt and sham groups did have local internal diameter reductions in the acinar and lobular arteries $(-1.1 \% \pm 1.0 \%$ in the anastomosed side vs $-17.7 \% \pm$ $3.5 \%$ in the nonanastomosed side vs $-20.9 \% \pm 6.1 \%$ in the sham group; $P=.03$ ). In the morphometric studies, the internal diameter of the pulmonary artery accompanying the terminal bronchiole in the anastomosed side of the cavopulmonary shunt group was significantly larger, and the ratio of medial thickness relative to the outer diameter was smaller compared with ratios in the nonanastomosed side of the cavopulmonary shunt group and the sham group.

Conclusions: We developed a rabbit cavopulmonary shunt model. In the anastomosed side of the cavopulmonary shunt group, the peripheral pulmonary arteries, which contributed greatly in regulating the pulmonary vascular resistance, had a local reduction in the basal vascular tone and no hypoxic vasoconstriction 2 weeks after the operation. 


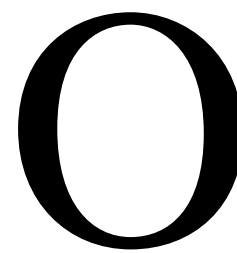

ver the past decade, bidirectional cavopulmonary shunt (CPS) has become a standard staging procedure in younger infants and has resulted in improved outcomes of right heart bypass. ${ }^{1,2}$ However, the appearance of desaturation, which is a symptom of pulmonary arteriovenous malformation (PAVM), has been reported in patients who underwent bidirectional CPS in their early infancy. ${ }^{3-6}$ PAVM is a widely known complication that causes significant problems in some patients after a CPS operation. ${ }^{7-9}$ With conventional angiography, the PAVM is detectable as a small, diffuse angiomatoid formation. ${ }^{6,9}$ PAVM in historical studies, however, has presented with dilated and clustered peripheral pulmonary vessels in the lung after CPS. ${ }^{9,10}$ Several studies of echocardiography have shown an increase in the right-to-left shunt by $60 \%$ in patients after CPS despite the absence of clinical features of PAVM. ${ }^{5,6}$ A radionuclide study $^{11}$ among patients after CPS showed that PAVM is regarded as a universal phenomenon. Judging from these previous studies of PAVM, ${ }^{5-10}$ it has been shown that pulmonary artery (PA) remodeling, such as vasodilation or the lack of vasoconstriction, might occur after CPS.

Despite the extensive analyses of the PA after CPS, the physiologic characteristics of the PA after CPS have not been thoroughly investigated. In this study, to evaluate the changes of the physiologic characteristics of the PA after CPS, we developed a small-animal CPS model by using rabbits. By applying a specially designed $\mathrm{x}$-ray television system, ${ }^{12}$ which is able to visualize small arteries with high resolution, we measured the internal diameter (ID) of the acinar, lobular (resistance), and segmental (conduit) PA. Moreover, this study focused on the hypoxic pulmonary vasoconstriction response, which is essential for regulating the pulmonary vascular tone while optimizing gas exchange in the lung by matching the rate of exchange between ventilation and perfusion. We then analyzed the changes in the basal tone and the hypoxia response after CPS along each vascular level.

\section{Methods}

This protocol was approved by the Institutional Animal Care and Use Committee of Kyoto University. All animals received humane care in compliance with the "Guide for the Care and Use of Laboratory Animals" prepared by the Institute of Laboratory Animal Resources, National Research Council, and published by the National Academy Press, revised 1996.

\section{Cavopulmonary Shunt}

Japanese white rabbits (12 to 16 weeks of age and weighing 2.2 to $2.9 \mathrm{~kg}$ ) were used as a CPS model. A 24-gauge intravenous catheter was placed in a marginal ear vein for intravenous access. Anesthesia was induced by intravenous pentobarbital sodium 25 $\mathrm{mg} / \mathrm{kg}$. The rabbits were then orally intubated and mechanically ventilated (Harvard Apparatus Constant Volume Ventilator model 683; Harvard Apparatus Co, South Natick, Mass). Anesthesia was maintained with $1.5 \%$ isoflurane. A warming pad was used to prevent hypothermia. Ringer's lactate was infused at $10 \mathrm{~mL} \cdot \mathrm{kg}^{-1}$ $\cdot \mathrm{h}^{-1}$. After median sternotomy in a supine position, the right superior vena cava (SVC) was extensively dissected, and all tributaries of the SVC were ligated. Great care was taken not to injure the right phrenic nerve. After heparin sodium $1 \mathrm{mg} / \mathrm{kg}$ was administered intravenously, the SVC was ligated at the junction to the right atrium with 6-0 polypropylene sutures. After dissection of the right $\mathrm{PA}$, the SVC was anastomosed to the right PA in an end-toside fashion with 8-0 polypropylene sutures. Great care was taken to prevent stenosis at the site of anastomosis, because this results in the death of the animal. In preliminary studies, we performed macroangiography to confirm that the anastomosed site was without stenosis. The right PA was ligated proximally to the anastomosed site (Figure 1). The left SVC was ligated close to the coronary vein. After these procedures, the chest was closed with a mediastinal drainage tube in place, and then extubation was performed after the rabbits were fully awakened from anesthesia. In rabbits in the sham group, the right SVC and right PA were dissected and clamped for 10 minutes under heparinization, as described previously, without making a CPS.

\section{Pulmonary Microangiography}

The rabbits were subjected to pulmonary microangiography 2 weeks after the operation. Anesthesia was induced by intravenous pentobarbital sodium $30 \mathrm{mg} / \mathrm{kg}$ and was maintained with pentobarbital sodium $2.5 \mathrm{~mL} \cdot \mathrm{kg}^{-1} \cdot \mathrm{h}^{-1}$. The rabbits were then orally intubated and mechanically ventilated as described previously. Two catheters were inserted into the right femoral artery and vein to measure the arterial pressure and to inject drugs, respectively. The bilateral rib cages were partially excised between the fourth and eighth intercostal spaces, and the pleura were opened to expose the lower lobe of each lung. A catheter, for injection of contrast medium, was introduced from the right jugular vein into the right PA via the CPS. Another catheter was introduced to the left PA via the right ventricle.

The system and the experimental setup used for angiography have been described previously in detail. ${ }^{12}$ In brief, the rabbit was placed inside an x-ray apparatus box (Hitex, Osaka, Japan) and fixed just above the beryllium faceplate of an x-ray-sensitive vidicon camera (Hamamatsu Photonics, Hamamatsu, Japan). During a temporary cessation from the ventilation at end-expiration, contrast medium $(2.7 \mathrm{~mL} ; 60 \%$ meglumine diatrizoate) was injected into each PA at a constant speed $(1.5 \mathrm{~mL} / \mathrm{s})$, and its passage through the pulmonary vascular bed was recorded serially with a videotape recorder (model PVW-2800; Sony, Tokyo, Japan).

To determine the ID of the PA, 4 serial frames of the angiographic images were added and then averaged by the digital image processor (DVS-5000; Hamamatsu). The processed image was electrically transferred to an image hard copy unit (model UP-960; Sony) and copied clearly onto paper. The pulmonary vascular ID on the copy was then measured with a digitizer (model 9874A; Hewlett-Packard, Palo Alto, Calif) connected to a minicomputer. After the method described in our previous study, ${ }^{13}$ ID measurements were made from the random selection of at least 5 branches of each vascular site, which were classified into 3 vascular groups 


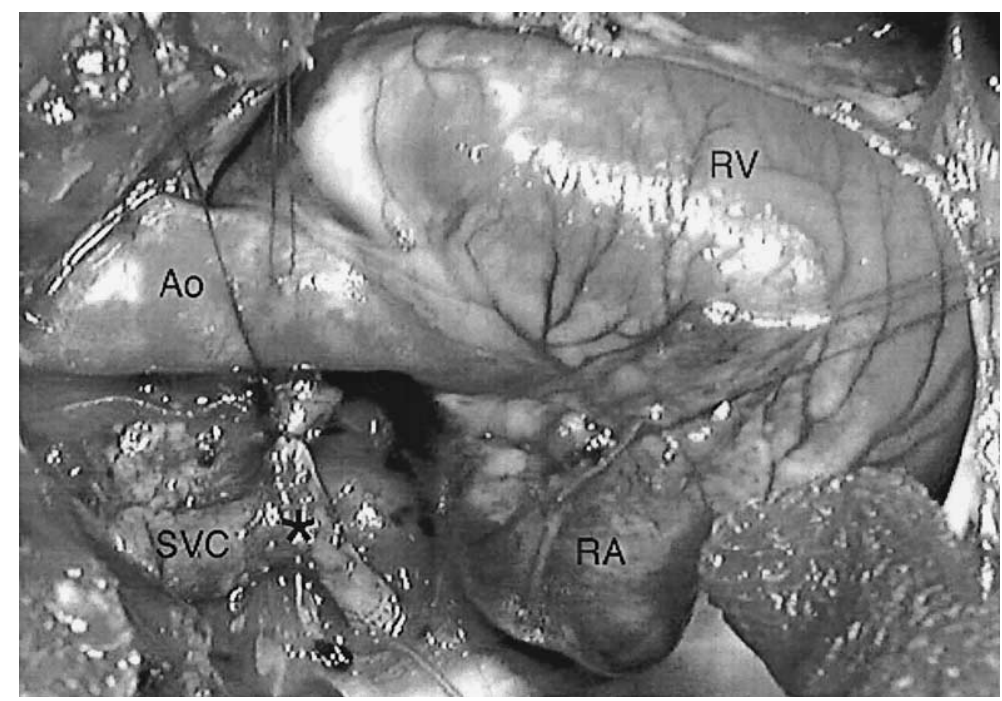

Figure 1. Operative schema after cavopulmonary anastomosis of the right superior vena cava to the right pulmonary artery and ligation of the right pulmonary artery proximally to the site of cavopulmonary anastomosis. $A o$, Aorta; RV, right ventricle; $R A$, right atrium; $S V C$, superior vena cava.

(ie, acinar-, lobular-, and segmental-level PAs). The mean values of the ID were then obtained in each group.

The ID response to hypoxia was examined. The pretreatment angiography was performed, and then hexamethonium bromide 8 $\mathrm{mg} / \mathrm{kg}$ (Sigma Chemical Co, St Louis, Mo) and atropine sulfate 7 $\mathrm{mg} / \mathrm{kg}$ (Sigma Chemical Co) were intravenously injected to suppress the influence of autonomic nerve activity changes during hypoxia. After the treatment, the baseline angiogram was recorded. There were no significant differences in IDs between pretreatment and baseline angiograms. Thereafter, rabbits were ventilated with $8 \%$ oxygen for 3 minutes, and the angiogram was recorded at the end of hypoxic ventilation. Hemodynamic data were also measured before and during hypoxia.

\section{Histologic Evaluation of the Lung}

After the angiography, rabbits were killed with an overdose of pentobarbital sodium. Paraformaldehyde $4 \%$ was infused through the tracheal tube at a pressure of $20 \mathrm{~cm} \mathrm{H}_{2} \mathrm{O}$. After the fixation, both lungs were excised and immersed in $4 \%$ paraformaldehyde at least 24 hours. A block of the lower lobe, which corresponds to the site of microangiography, was cut out from each lung. These blocks were embedded in paraffin, and $4-\mu \mathrm{m}$ slices were subjected to hematoxylin-eosin and elastica van Gieson stains. The sliced lungs were then examined by a light microscope, which was connected to a computer-assisted image analysis system (VIP$21 \mathrm{CH}$ color video image processor; Ikegami, Kawasaki, Japan). In each slice, 10 randomly selected cross-sectioned resistance arteries accompanying terminal bronchioles were identified. The circumference of the internal elastic lamina was measured and divided by $\pi$ to determine the ID of the arteries. The medial thickness (MT) of the arteries was determined by the average of the MT in each quarter slice of artery. The ratio of MT to the outer diameter of the artery was determined as follows: $2 M T /(2 M T+I D)$. The mean values of each parameter were used for comparison.

\section{Statistical Methods}

All values are expressed as a mean \pm SE. The Mann-Whitney $U$ test was used to compare among 3 groups: the right (anastomosed) side and the left (nonanastomosed) side of the CPS group and the sham group. The Wilcoxon signed-rank test was assigned to study the responses from each group to compare those with and without hypoxia.

\section{Results}

\section{Blood Gas Analysis}

Table 1 represents the results of blood gas analysis at 2 weeks after operation. Before we collected the blood samples, we confirmed normal movements of the bilateral diaphragm and no massive atelectasis or pleural effusion affected blood gas exchange by thoracoscopy. The oxygen tension of the arterial blood for the CPS group was significantly lower than that of the sham group $(68.2 \pm 2.2 \mathrm{~mm}$ $\mathrm{Hg}$ vs $91.1 \pm 1.9 \mathrm{~mm} \mathrm{Hg} ; P=.01)$.

\section{Hemodynamic Data}

The mean left PA pressure (PAP), mean right PAP via CPS, and mean systemic arterial pressure under baseline and hypoxic conditions are summarized in Table 2 . The mean left PAP of both groups increased in response to hypoxia (sham group: $3.5 \mathrm{~mm} \mathrm{Hg}, P=.04$; CPS group: $3.0 \mathrm{~mm} \mathrm{Hg}$, $P=.06)$. In contrast, the mean right PAP of the CPS group remained at the baseline level.

\section{Arteriogram From Both Lungs of CPS and Sham Rabbits}

Figure 2 shows typical arteriograms before (Figure 2, $A$ and $B$ ) and during (Figure 2, $C$ and $D$ ) hypoxia from the lung of 
TABLE 1. Characterization of postoperative cavopulmonary shunt model

\begin{tabular}{lcc}
\hline Variable & Sham & CPS \\
\hline $\mathrm{n}$ & 4 & 5 \\
Body weight $(\mathrm{kg})$ & $2.6 \pm 0.1$ & $2.7 \pm 0.1$ \\
Arterial blood gases & & \\
$\quad \mathrm{pH}$ & $7.37 \pm 0.02$ & $7.39 \pm 0.02$ \\
$\quad \mathrm{PcO}_{2}$ & $35.3 \pm 1.0$ & $39.1 \pm 1.3^{*}$ \\
$\mathrm{PO}_{2}$ & $91.1 \pm 1.9$ & $68.2 \pm 2.2 \dagger$ \\
\hline
\end{tabular}

CPS, Cavopulmonary shunt; $\mathrm{PO}_{2}$ and $\mathrm{PCO}_{2}$, oxygen tension and carbon dioxide tension of arterial blood, respectively. Values are mean $\pm \mathrm{SE}$.

${ }^{*} P=.03$; significant difference from sham.

$\mathrm{\dagger} P=.01$; significant difference from sham.

the anastomosed side of the CPS group and the sham group. In the anastomosed side of the CPS group (Figure 2, A), the baseline IDs increased (particularly in the lobular and acinar levels) when compared with those for the sham group (Figure 2,B). In the sham group (Figure 2,D), the IDs of the lobular- and the acinar-level PAs, but not the segmentallevel PAs, decreased in response to hypoxia. The IDs of the anastomosed side of the CPS group exhibited no hypoxic change in any level of the arteries (Figure 2, C).

The mean IDs of the PA were compared among the anastomosed and nonanastomosed sides of the CPS group and the sham group, as shown in Figure 3. The IDs of the lobular- and acinar-level PAs of the anastomosed side of the CPS group were significantly larger than those of the nonanastomosed side and the sham group. Among these 3 lungs in the segmental level PAs, however, there were no significant differences; this means that the local baseline ID increased in the lobular and acinar levels of the anastomosed side of the CPS group.

Figure 4 shows the mean value of percentage ID changes in response to $8 \%$ oxygen inhalation in the bilateral lungs of the CPS group and the sham group. In the nonanastomosed side of the CPS group and the sham group, the ID of lobular- and acinar-level, but not segmental-level, arteries was significantly reduced in response to hypoxia. In contrast, such a hypoxic response did not occur in any of the arterial levels of the anastomosed side of the CPS group.

\section{Morphometric Measurement}

Figure 5 shows the typical resistance vessels accompanying the end of the terminal bronchiole in bilateral lungs of the CPS group and the sham group. The results of the morphometric measurements are summarized in Table 3. The ID was larger in the anastomosed side of the CPS group when compared with the nonanastomosed side of the CPS group and the sham group. These results were consistent with the findings of the microangiogram. In the sham group, there was no significant difference between bilateral lungs (data not shown). The MT of resistance arteries in the anasto-
TABLE 2. Hemodynamic data

\begin{tabular}{lccc}
\hline Variable & Left PAP $(\mathbf{m m} \mathbf{~ H g})$ & SAP $(\mathbf{m m ~ H g})$ & Right PAP $(\mathbf{m m ~ H g})$ \\
\hline Sham $(\mathrm{n}=4)$ & & & \\
Baseline & $16.5 \pm 1.6$ & $72 \pm 4$ & Not determined \\
Hypoxia & $20.0 \pm 1.4^{*}$ & $70 \pm 4$ & Not determined \\
CPS $(\mathrm{n}=5)$ & & & \\
Baseline & $19.0 \pm 1.3$ & $76 \pm 10$ & $8.0 \pm 0.3$ \\
Hypoxia & $22.0 \pm 1.3$ & $73 \pm 8$ & $8.0 \pm 0.3$
\end{tabular}

$P A P$, Mean pulmonary artery pressure; $S A P$, mean systemic artery pressure; $C P S$, cavopulmonary shunt. Values are a mean $\pm \mathrm{SE}$.

${ }^{*}$ Significant difference $(P=.05)$ from baseline.

mosed side of the CPS group was thinner than in the sham group and in the nonanastomosed side of the CPS group.

\section{Discussion}

Using our $\mathrm{x}$-ray television system $^{12}$ on the rabbit CPS model in vivo, we have shown dilatation of the acinar and lobular (resistance) arteries in the anastomosed side of the CPS group under baseline conditions. In contrast, the segmental arteries remained unchanged. In terms of clinical angiography, ID changes in the peripheral resistance arteries, however, could not be measured quantitatively because of the resolution limitation. In this study, we clearly demonstrated the local dilatation of the acinar- and lobular-level arteries in the CPS group.

Our morphometric study showed consistent results that the peripheral pulmonary resistance arteries accompanying the terminal bronchiole had dilated, and the media illustrated that these arteries had become thin. Among patients with PAVM after CPS, similar histologic findings have been reported. ${ }^{9,10}$ Although our model did not show the development of PAVM, our data do support these previous findings ${ }^{9,10}$ with respect to the presence of pulmonary vasodilatation after a CPS operation.

We previously reported that alveolar hypoxia, per se, induced vasoconstriction (namely, within the resistance PAs through an intrinsic pulmonary mechanism ${ }^{13}$ of the cat) and that this locally induced hypoxic response was modulated by neural reflex effects during a global alveolar hypoxic event. ${ }^{14,15}$ Moreover, we have shown that the PA constriction during a vagus nerve stimulation was diminished by hexamethonium bromide or atropine sulfate in the rabbit ${ }^{16}$ and that the pulmonary sympathetic nerve activity disappeared with injection of hexamethonium bromide in the cat. ${ }^{15}$ Because this study focused on hypoxic pulmonary vasoconstriction, we also simultaneously administered hexamethonium bromide and atropine sulfate to suppress the secondary influence of the neural reflex changes before exposing global hypoxia. Under these pretreated conditions, global hypoxia selectively constricted the resistance PAs in the nonanastomosed side of the CPS group and the sham 

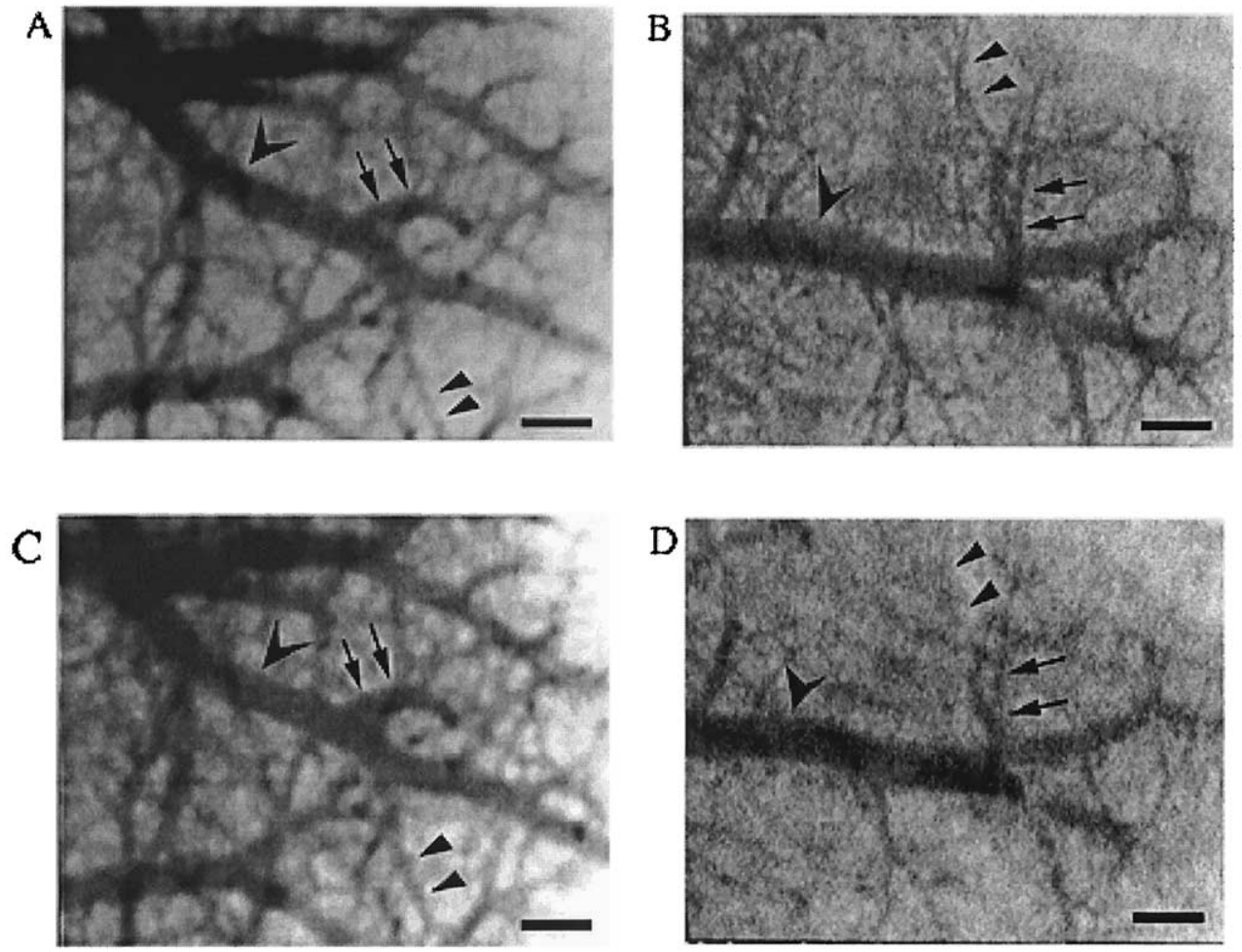

Figure 2. Typical angiograms of small pulmonary arteries before and during hypoxia (8\% oxygen). Left (A and C): the anastomosed side of the CPS group. Right (B and D): sham. Top (A and B): before hypoxia. Bottom (C and D): during hypoxia. In the sham group, lobular-level (arrow) and acinar-level (triangle) arteries constricted in response to hypoxia, but larger arteries did not (arrowhead). In the anastomosed side of the CPS group, no level of arteries responded. Black bar indicates $1000 \mu \mathrm{m}$.

group (Figures 2 and 3); this is consistent with the site of the locally induced hypoxic vasoconstriction. ${ }^{13}$

This study showed that no hypoxic response of resistance arteries was induced in the anastomosed side of the CPS group, which had nonpulsatile flow, and no blood from the inferior vena cava was shown. It is generally agreed that under hypoxia, resistance arteries induce an increase in flow resistance within the hypoxic lung. ${ }^{17,18}$ Even if the lung were perfused with an acute nonpulsatile constant flow, the pressure response to hypoxia would remain unchanged. ${ }^{19}$ Because hypoxic vasoconstriction did occur in the nonanastomosed side that was perfused with inferior vena cava blood, in turn, unresponsiveness to hypoxia might be due to the lack of inferior vena cava blood. It is interesting to note that pulmonary circulatory abnormalities in the rat model with liver dysfunction were manifested by the vasodilation of the peripheral pulmonary vessels and blunted hypoxic vasoconstriction. ${ }^{20}$ Such abnormalities in the pulmonary vessel properties are very similar to the present data obtained in the anastomosed side of the CPS group. Although we could not find the cause of the disappearance of the response, this is still the first report that no hypoxic response in the resistance arteries was induced after CPS.
Although the fundamental mechanism of hypoxic pulmonary vasoconstriction is not clearly defined, it is an intrinsic property of the PA smooth muscle cell itself. It has also been shown that isolated PAs need pretone induced by some vasoactive agents to constrict in response to hypoxia. ${ }^{21}$ Malhotra and colleagues ${ }^{22}$ have reported a reduction of angiotensin-converting enzyme in the lung and a decrease of circulating levels of angiotensin II after CPS. Moreover, in the rat liver cirrhosis model, which has blunted hypoxic pulmonary vasoconstriction, the reduction of endothelin-1 in the lung has been documented. ${ }^{23}$ Although we could not measure angiotensin II and endothelin-1 in our study, the change of these vasoactive agents in the lung might contribute to the lack of hypoxic response and resistance PA vasodilation. Further study is needed to evaluate this mechanism.

Arterial oxygen pressure in the CPS animals, without confirming obvious development of PAVM, was significantly lower than in the sham animals. We confirmed that there was no existence of massive atelectasis in the lung or abnormal movement of the diaphragm, and the following factors may explain this mechanism. The first factor is the abnormal dilatation of the peripheral resistance arteries. It 


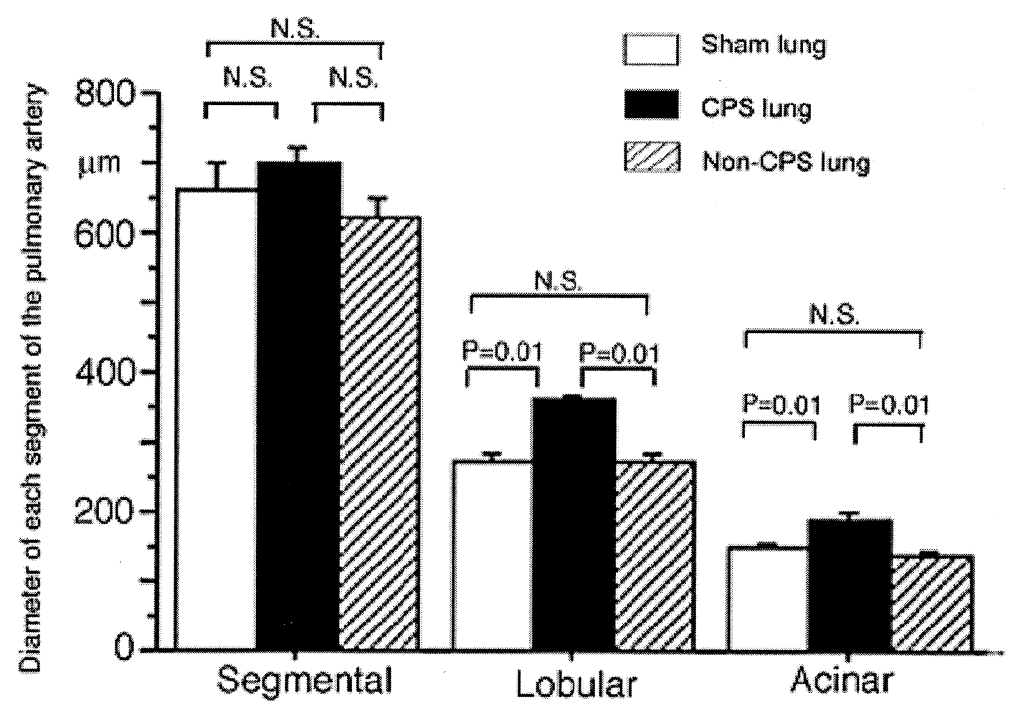

Figure 3. Comparison of internal diameter under baseline conditions among sham group lungs and the right (anastomosed) lungs and left (nonanastomosed) lungs of the CPS group. The baseline internal diameter of the anastomosed-side lung was larger than those of other lungs in lobular- and acinar-level arteries, but not larger than in segment-level arteries. Black bar $(n=5)$ : the anastomosed side of the CPS group. Hatched bar $(n=5)$ : the nonanastomosed side of the CPS group. White bar $(n=4)$ : sham group. N.S., Not significant.

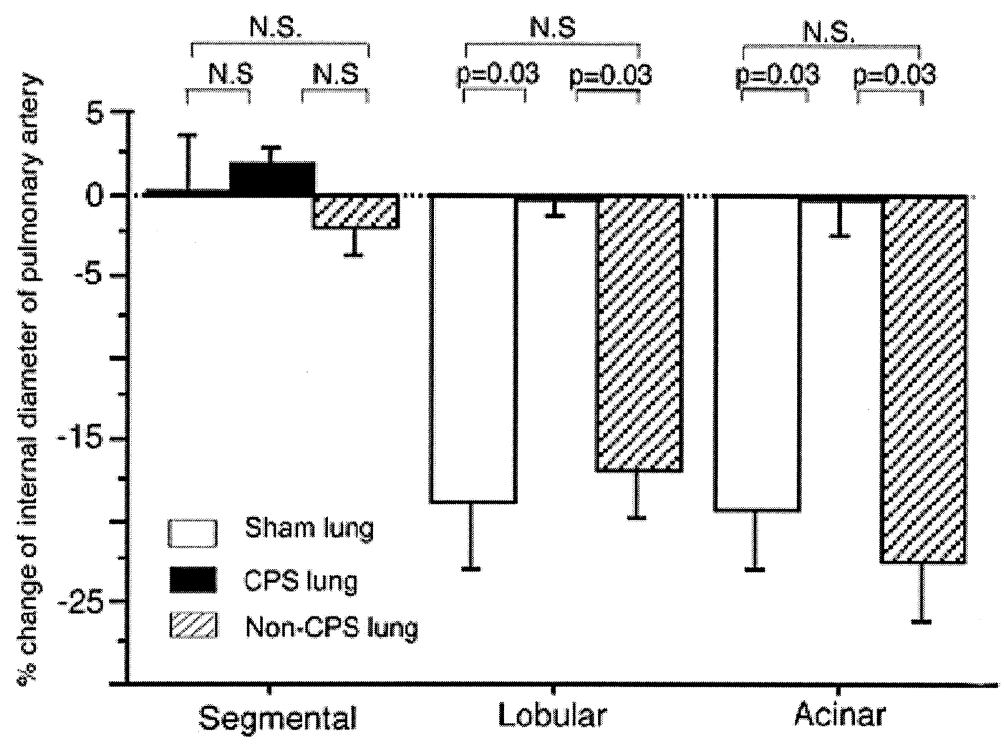

Figure 4. Comparison of percentage changes in ID in response to hypoxia (8\% oxygen) among left (nonanastomosed) and right (anastomosed) lungs of the CPA and sham group lungs. Hypoxic ID reduction was not induced in the anastomosed lung but did occur in other lungs. Black bar $(\mathbf{n}=5)$ : the anastomosed side of the CPA group. Hatched bar $(\mathrm{n}=5)$ : the nonanastomosed side of the CPS group. White bar $(\mathrm{n}=4)$ : sham group. N.S., Not significant.

has been shown that the nonpulsatile passive return of blood to the lungs after a CPS causes more dominant flow into the lower lobes, ${ }^{24}$ making a mismatch between ventilation and perfusion. Furthermore, abnormal vasodilatation without hypoxic pulmonary vasoconstriction may further enhance this mismatch. In a long-term study in a goat model, with a nonpulsatile pulmonary perfusion operated by a centrifugal pump, the blood distribution in the pulmonary circulation and the blood gas contents did not change. ${ }^{25}$ The lack of hepatic venous blood might be a contributing factor in the 

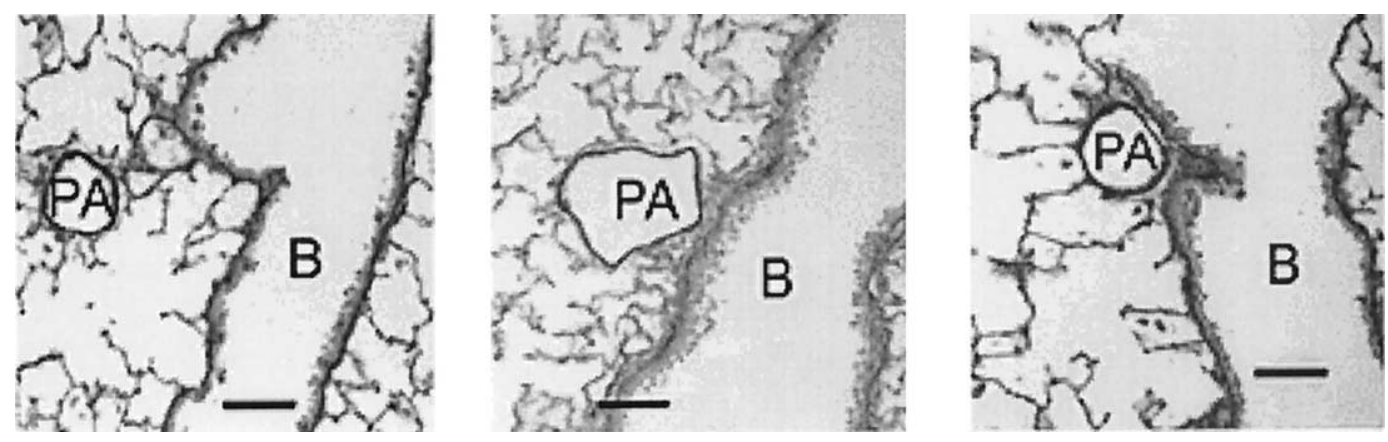

Figure 5. Typical light microscopic photography of small pulmonary arteries in the end of a terminal bronchiole 2 weeks after the operation. Left: sham group. Center: the anastomosed side of the CPS group. Right: the nonanastomosed side of the CPS group. The pulmonary artery of the anastomosed side of the CPS group was dilated larger than in the other 2 groups. PA, Small pulmonary artery; B, bronchiole. Black bar indicates $100 \mu \mathrm{m}$.

TABLE 3. Morphometry of resistance vessels

\begin{tabular}{lccc}
\hline & & \multicolumn{2}{c}{ Cavopulmonary shunt } \\
\cline { 3 - 4 } Variable & Sham & $\begin{array}{c}\text { Nonanastomosed } \\
\text { side }\end{array}$ & $\begin{array}{c}\text { Anastomosed } \\
\text { side }\end{array}$ \\
\hline $\begin{array}{l}\text { Internal diameter } \\
(\mu \mathrm{m})\end{array}$ & $104 \pm 2$ & $115 \pm 9$ & $169 \pm 13^{*} \dagger$ \\
$\begin{array}{c}\text { Medial thickness } \\
(\mu \mathrm{m})\end{array}$ & $6.1 \pm 0.5$ & $5.7 \pm 0.5$ & $4.8 \pm 0.2 \ddagger$ \\
$\% \mathrm{MT}(\%)$ & $10.5 \pm 0.7$ & $9.3 \pm 1.2$ & $5.5 \pm 0.5^{*} \dagger$
\end{tabular}

$\% M T$, Percentage of medial thickness, calculated by the following formula: medial thickness $\times 2$ /medial thickness $\times 2+$ internal diameter.

*Significant difference $(P=.01)$ from sham.

tSignificant difference $(P=.01)$ from the nonanastomosed side.

¥Significant difference $(P=.03)$ from sham and the nonanastomosed side.

maldistribution of pulmonary blood flow rather than a lack of pulsatility, as a result. Another possible factor might be the lack of hypoxic pulmonary vasoconstriction in the peripheral resistance arteries under CPS. If the latter possibility were to occur, it could actually aggravate the mismatch between ventilation and perfusion. It is not yet known whether the vascular tone could become abnormal even under normoxic conditions, and further study is necessary to investigate the influence of flow pulsatility to the pulmonary circulation.

In terms of anesthetic drugs, we used pentobarbital sodium, which has an effect on vasodilation, for the induction of anesthesia in both groups. However, we can disregard the effect of this drug because the difference in IDs between the anastomosed side and the nonanastomosed side was evident. Because the life span of rabbits is different from that of humans, the changes in the time course of pulmonary vascular tone regulation after a CPS operation in rabbits may not correspond to those of humans. Likewise, in terms of progress in the time course of vascular remodeling, there might be a difference between these 2 species. It might be necessary to further study the changes in the structure and function of the pulmonary vasculature during the earlier and later stages of the rabbit CPS model. Another limitation is that clear angiograms of the pulmonary veins could not be obtained in the CPS lung. This is because the blood-flow velocity in the abnormally dilated arteries was very slow. In terms of histologic study, the lungs were not perfusion-fixed at a standard pressure. Therefore, it is difficult to compare differences in PA wall thickness and muscular development between animals.

In conclusion, we developed a new small-animal CPS model by using rabbits for this study. Our findings showed that the lobular-level and the acinar-level resistance arteries were abnormally dilated and had no hypoxic vasoconstriction within 2 weeks after a CPS operation was performed. This model, as a result, will enable us to evaluate the changes in the time course of the pulmonary vascular characteristics after the CPS operation.

\section{References}

1. Bridges ND, Jonas RA, Mayer JE, Flanagan MF, Keane JF, Castaneda AR. Bidirectional cavopulmonary anastomosis as interim palliation for high risk Fontan candidates. Early results. Circulation. 1990;82(5 suppl):IV170-6.

2. Reddy VM, Liddicoat JR, Hanley FL. Primary bidirectional superior cavopulmonary shunt in infants between 1 and 4 months of age. Ann Thorac Surg. 1995;59:1120-6.

3. Bacha EA, Jonas RA, Mayer JE Jr, Perry S, del Nido PJ. Management of pulmonary arteriovenous malformations after surgery for complex congenital heart disease. J Thorac Cardiovasc Surg. 1999;119:175-6.

4. Bernstein H, Brok MM, Silverman NH, Bristow J. Development of pulmonary arteriovenous fistulae in children after cavopulmonary shunt. Circulation. 1995;92(9 suppl):II309-14.

5. Chang R, Alejos JC, Atkinson D, Atkinson D, Jensen R, Drant S, et al. Bubble contrast echocardiography in detecting pulmonary arteriovenous malformation in children with univentricular heart after cavopulmonary anastomosis. J Am Coll Cardiol. 1999;33:2052-8.

6. Bernsein HS, Ursell PC, Brook MM, Hanley FL, Silverman NH, Bristow J. Fulminant development of pulmonary arteriovenous fistulas in an infant after total cavopulmonary shunt. Pediatr Cardiol. 1996; 17:46-50. 
7. Cloutier A, Ash JM, Smallhorn JF, Williams WG, Trusler GA, Rowe $\mathrm{RD}$, et al. Abnormal distribution of pulmonary blood flow after the Glenn shunt or Fontan procedure: risk of development of arteriovenous fistulae. Circulation. 1985;72:471-9.

8. Trusler GA, Williams WG, Cohen AJ, Rabinovitch M, Moes CA, Smallhorn JF, et al. William Glenn lecture. The cavopulmonary shunt. Evolution of a concept. Circulation. 1990;82(5 suppl):IV131-8.

9. Srivastava D, Preminger T, Lock JE, Mandell V, Keane JF, Mayer JE $\mathrm{Jr}$, et al. Hepatic venous blood and the development of pulmonary arteriovenous malformations in congenital heart disease. Circulation. 1995;92:1217-22.

10. Duncan BW, Kneebone JM, Chi EY, Hraska V, Isik FF, Rosenthal GL, et al. A detailed histologic analysis of pulmonary arteriovenous malformations in children with cyanotic congenital heart disease. J Thorac Cardiovasc Surg. 1999;117:931-8.

11. Vettukattil JJ, Slavik Z, Lamb RK, Monro JL, Keeton BR, Tsang VT, et al. Intrapulmonary arteriovenous shunting may be a universal phenomenon in patients with the superior cavopulmonary anastomosis: a radionuclide study. Heart. 2000;83:425-8.

12. Sada K, Shirai M, Ninomiya I. X-ray TV system for measuring microcirculation in small pulmonary vessels. J Appl Physiol. 1985;59: 1013-8.

13. Shirai M, Sada K, Ninomiya I. Effect of regional alveolar hypoxia and hypercapnia on small vessels in cats. J Appl Physiol. 1986;61:440-8.

14. Shirai M, Shindo T, Ninomiya I. Beta-adrenergic mechanisms attenuated hypoxic pulmonary vasoconstriction during systemic hypoxia in cats. Am J Physiol. 1994;266:H1777-85.

15. Shirai M, Matsukawa K, Nishiura N, Kawaguchi AT, Ninomiya I. Changes in efferent pulmonary sympathetic nerve activity during systemic hypoxia in anesthetized cats. Am J Physiol. 1995;269: R1404-9.

16. Sada K, Shirai M, Ninomiya I. Vagally and acetylcholine-mediated constriction in small pulmonary vessels of rabbits. J Appl Physiol. 1987;63:1601-9.

17. Dawson CA. Role of pulmonary vasomotion in physiology of the lung. Physiol Rev. 1984;64:544-616.

18. Grover RF, Wagner WW Jr, McMurtry IF, Reeves JT. Pulmonary circulation. In Shepherd JT, Abboud FM, editors: Handbook of physiology. The cardiovascular system. Peripheral circulation and organ blood flow, vol 3, sect 2. Bethesda (MD): American Physiological Society; 1983. p. 103-36.

19. Gregory TJ, Ellsworth ML, Newell JC. Hypoxic pulmonary vasoconstriction during steady and pulsatile flow in ferrets. J Appl Physiol. 1984;57:205-12.

20. Chang S, Ohara N. Pulmonary circulatory dysfunction in rats with biliary cirrhosis: an animal model of the hepatopulmonary syndrome. Am Rev Respir Dis. 1992;145(4 Pt 1):798-805.

21. Leach RM, Robertson TP, Twort CH, Ward JP. Hypoxic vasoconstriction in rat pulmonary and mesenteric arteries. Am J Physiol. 1994; 266(3 Pt 1):L223-31.

22. Malhotra SP, Riemer RK, Thelitz S, He YP, Hanley FL, Reddy VM. Superior cavopulmonary anastomosis suppresses the activity and expression of pulmonary angiotensin-converting enzyme. J Thorac Cardiovasc Surg. 2001;122:464-9.

23. Carter EP, Sato K, Morio Y, McMurtry IF. Inhibition of $\mathrm{K}(\mathrm{Ca})$ channels restores blunted hypoxic pulmonary vasoconstriction in rats with cirrhosis. Am J Physiol Lung Cell Mol Physiol. 2000;279:L90310 .

24. Laks H, Mudd JD, Standeven JW, Fagan JW, Willman VL. Long-term effect of the superior vena cava-pulmonary anastomosis on pulmonary blood flow. J Thorac Cardiovasc Surg. 1977;74:253-60.

25. Sakaki M, Taenaka Y, Tatsumi E, Nakatani T, Takano H. Influences of nonpulsatile pulmonary flow on pulmonary function: evaluation in a chronic animal model. J Thorac Cardiovasc Surg. 1994;108:495502 . 